\title{
An efficient and novel FDTD method based performance investigation in high-speed current-mode signaling SWCNT bundle interconnect
}

\author{
YASH AGRAWAL ${ }^{1, * D}$, M GIRISH $^{2}$ and RAJEEVAN CHANDEL $^{3}$ \\ ${ }^{1}$ VLSI and Embedded Systems Research Group, Dhirubhai Ambani Institute of Information and \\ Communication Technology (DA-IICT), Ganghinagar 382 007, India \\ ${ }^{2}$ Department of Electronics and Communication Engineering, Vidya Jyoti Institute of Technology, \\ Hyderabad 500 075, India \\ ${ }^{3}$ Department of Electronics and Communication Engineering, National Institute of Technology Hamirpur, \\ Hamirpur 177 005, Himachal Pradesh, India \\ e-mail:mr.yashagrawal@gmail.com; giri.frds@gmail.com; rchandel@ nith.ac.in
}

MS received 9 February 2016; revised 24 September 2017; accepted 27 July 2018; published online 12 September 2018

\begin{abstract}
Carbon nanotube (CNT) has emerged as the most extensively researched area in nanoscience and amongst the frontrunners in co-triggering the nanotechnology revolution. Single-wall CNT (SWCNT) bundle is a part of CNT family and has been proposed as the future nano-wires in integrated circuits. The present paper analyzes the performance of SWCNT bundle interconnect with high-speed current-mode signaling (CMS) scheme using efficient finite-difference time-domain (FDTD) method. For the first time, FDTD based method is explored for modeling CMS SWCNT bundle interconnect incorporating practical CMOS driver gate. The CMOS gate is characterized by $\mathrm{n}^{\text {th }}$ power-law model. The stability of FDTD method is ascertained by Courant condition. The proposed FDTD based method is efficient and can be used for performance analyses of future nano-wire SWCNT bundle as well as conventional copper interconnects. At the same time, this method is applicable for both traditional full-voltage swing voltage-mode signaling (VMS) and remarkable low-voltage swing CMS schemes. The various analyses in the paper reveal that CMS SWCNT bundle interconnect has higher edge over CMS copper interconnect in terms of smaller delay, lesser crosstalk induced delay and noise. The proposed analytical FDTD based method is validated using Tanner-SPICE EDA simulation tool. The maximum error between the FDTD and SPICE for the transient response in CMS SWCNT bundle interconnect for $32 \mathrm{~nm}$ technology node is within $3 \%$.
\end{abstract}

Keywords. Current-mode signaling (CMS); finite-difference time-domain (FDTD); nanotechnology; on-chip interconnect; single-wall carbon nanotube (SWCNT) bundle.

\section{Introduction}

Since the discovery of carbon-nanotube (CNT) in 1991, the remarkable, inimitable and unprecedented properties of CNT have arisen lots of research interest and excitement in nano field $[1,2]$. The CNTs are used in variety of promising different applications such as microelectronics/nanoelectronics [3], molecular electronics and quantum computing [4], optics [5], spintronics [6], material science [7], mechanical [8] and biological fields [9]. Depending on the topology, structure and dimensions, CNTs possess either metallic or semiconductive characteristics [10]. In the field of nanoelectronics, metallic CNTs have been proposed as a potential replacement to copper material for on-chip interconnections in integrated circuit due to its stupendous properties as insensitivity to electro-migration [11], ballistic carrier conduction [12], long electron mean free path

*For correspondence
[13], high mechanical and thermal stability [14] and high thermal conductivity [15].

CNTs can be broadly classified as single-wall CNT (SWCNT) and multi-wall CNT (MWCNT) [16]. SWCNT is a seamless cylinder of graphene whose diameter varies from $0.4 \mathrm{~nm}$ to $4 \mathrm{~nm}$. On the other hand, MWCNT comprises of multiple concentric shells that are bound together by weak interlayer Van der Waal's force [4]. The diameter of MWCNT ranges from several nanometers to tens of nanometer [17]. An isolated SWCNT has large parasitic impedance. In $[18,19]$, a comparison has been made between copper and monolayer SWCNT interconnects. It is reported that isolated SWCNTs are slower than their counterpart copper interconnects. To mitigate this limitation, a bundle of SWCNT is often used for on-chip interconnect applications. Owing to parallel arrangement, the overall SWCNT bundle interconnect impedance reduces. Furthermore, this enhances the number of conduction channels and hence current conduction [20]. In [18, 19, 21], it is investigated that the 
performance of bundle SWCNTs can outperform copper interconnect in terms of lesser circuit propagation delay. Further, it is shown that the performance of SWCNT bundle increases as the length of interconnect increases or the technology scales down [22]. In [10, 23, 24], crosstalk analysis has been performed and it is investigated that SWCNT bundle interconnect leads to reduced crosstalk noise and lesser crosstalk induced delay than copper interconnect. Das has found that in concern with gate oxide reliability, SWCNT bundle interconnect is more suitable than copper interconnect [25]. These investigations suggest that SWCNT bundle interconnect is certainly better than conventional copper interconnect and is the potential and prospective candidate to be used as future nano-wires.

From the literature it is seen that most of the researches have analyzed SWCNT bundle interconnect using simulation based models. However, no fundamental mathematical models have been formulated for the performance evaluation of these interconnects [10, 18-20, 22-26]. In [21], SWCNT bundle interconnect is approximated as $R C$ model and propagation delay is computed using Elmore based model. However, this model has limited accuracy as it captures only the dominant system poles in which the higher order moments of the system are neglected. Also, at high frequencies, inductance becomes significant and $R C$ interconnect model has limited significance. The model presented in [27], has considered numerical technique based finite-difference time-domain (FDTD) technqiue for the performance characterization of SWCNT bundle interconnect. This model has however ignored metal-nanotube contact resistance. The models demonstrated in [19, 21, 24, 27] have approximated CMOS driver gate as resistive and capacitive elements. This approximation however results in erroneous output since non-linear CMOS gate cannot be represented by single lumped value for different regions of operation of MOS transistors during transition period. Nevertheless, this issue is mitigated by incorporating a practical CMOS gate in the present analysis.

The signaling schemes in interconnect play a seminal role in enhancing the system performance. The traditional VMS scheme has full rail-to-rail swing whereas remarkable CMS scheme has reduced voltage swing over the interconnects [28, 29]. CMS scheme possesses peculiar properties as high speed [28, 30], high bandwidth [28], superior signal integrity [31] and high immune to electrostatic discharge (ESD) induced damage of MOS transistors [29]. The reduced voltage swing in CMS scheme is obtained by using specialized low input impedance current-mode receiver circuits [28, 29, 31, 32]. Until now, nearly all of the researches were focused on voltage mode signaling (VMS) scheme for SWCNT bundle interconnect [10, 18-27]. In [28, 31-34], the performance of copper interconnect using CMS scheme has been explored. It is investigated that CMS copper interconnect possesses high speed and bandwidth as compared to VMS copper interconnect. However, impressive current mode signaling (CMS) scheme has received very less attention and unfortunately meagerly explored for carbon based interconnects. This reveals that analysis and modeling of SWCNT bundle interconnect using CMS scheme has become extremely significant.

The present paper analyzes for the first time CMOS gate driven CMS SWCNT bundle interconnect using FDTD method. The proposed FDTD method is versatile as it can be applied to both copper and SWCNT bundle interconnects and concurrently to VMS and CMS schemes. The current section reviews the SWCNT bundle and CMS scheme for on-chip interconnects. The rest of the paper is organized as follows. Section 2 presents the electrical modeling of SWCNT bundle interconnect. In section 3, FDTD method based unified model for both copper and SWCNT bundle interconnects using VMS and CMS schemes is formulated. The results and discussion are presented in section 4. Finally, conclusions are provided in section 5 .

\section{Electrical modeling of SWCNT bundle interconnect}

The schematic of parallel SWCNT bundle interconnects is shown in figure 1. $w$ and $t$ represent the width and thickness of SWCNT bundle interconnect. $x$ denotes the interSWCNT distance. $s$ is the distance between two parallel SWCNT bundle interconnects. $h_{g}$ denotes the height above the ground plane and $d$ is the diameter of monolayer SWCNT. The unified model for copper and SWCNT bundle interconnects using VMS and CMS schemes is illustrated in figure 2. The interconnect is driven by CMOS gate and is same for both VMS and CMS schemes [10, 25-27, 31, 33, 34]. The receiver in VMS has high impedance termination. It is often designed using CMOS gate which is equivalently represented by load capacitance $\left(C_{L}\right)$ [10, 25-27]. In CMS scheme, low impedance termination is achieved by using specialized current mode receiver circuits $[28,29,32]$. It is equivalently modeled by parallel combination of load resistance $\left(R_{L}\right)$ and $C_{L}$ $[31,33,34]$. The value of $\alpha$ in figure 2 is ' 0 ' for copper leading to zero $R_{\text {lump }}^{\text {' }}$ resistance. It is ' 1 ' for SWCNT bundle interconnect. $\beta$ determines the signaling scheme. It is ' 0 ' for VMS and ' 1 ' for CMS scheme. The value of $\beta=0$, leads to infinite load resistance $\left(R_{L} / \beta\right)$ or open in case of VMS. The SWCNT bundle interconnect is represented by multiconductor transmission line model. This is simplified by using equivalent single conductor (ESC) model [35] and incorporated in the present analysis. The ESC SWCNT bundle interconnect model consists of parasitic resistance, inductance and capacitance. These parasitics along with their formulations are discussed in the following subsections.

\subsection{Number of SWCNTs in a bundle}

The number of SWCNTs along the width and thickness of the bundle interconnect are denoted as $N_{w}$ and $N_{t}$ respectively. These are obtained as [25] 


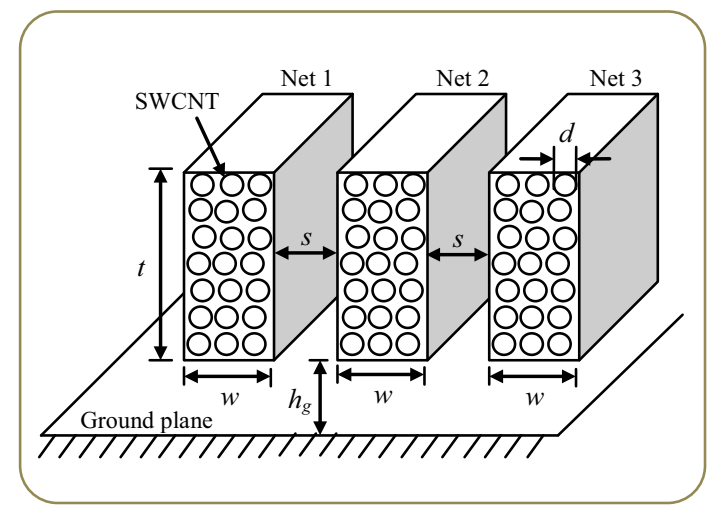

Figure 1. Schematic of parallel SWCNT bundle interconnect.

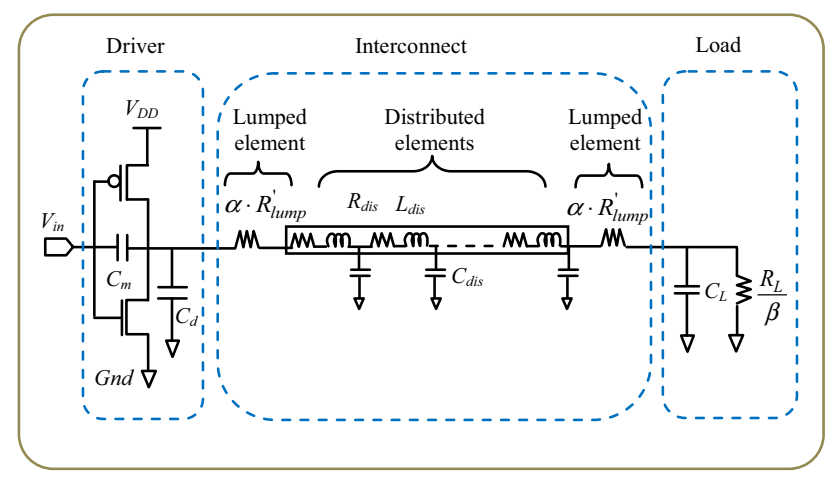

Figure 2. The unified model for CMOS gate driven copper and SWCNT bundle interconnect using VMS and CMS schemes.

$$
N_{w}=\operatorname{int}\left(\frac{w-d}{x}\right)
$$

and

$$
N_{t}=\operatorname{int}\left(\frac{2(t-d)}{\sqrt{3} x}+1\right)
$$

where int represents the integer value. In SWCNT bundle interconnect, $\left(P_{m}\right)$ denotes the metallic CNT ratio. $P_{m}$ varies between zero and unity [24]. In the present analysis, two particular cases of SWCNT bundle interconnect are considered viz. densely packed with $P_{m}=1$ and sparsely packed with $P_{m}=1 / 3$. The value of inter-CNT distance $x$ is $d$ and $\sqrt{3} d$ for the former and later, respectively [25].

The total number of SWCNTs $(N)$ in a bundle is computed as

$$
N=\left(\begin{array}{ll}
N_{w} N_{t}-\frac{N_{t}}{2} ; & \text { if } N_{t} \text { is even } \\
N_{w} N_{t}-\frac{\left(N_{t}-1\right)}{2} ; & \text { if } N_{t} \text { is odd }
\end{array}\right)
$$

\subsection{Resistance of SWCNT bundle interconnect}

The resistance of SWCNT bundle interconnect comprises of lumped and distributed elements. The lumped resistance constitutes contact resistance $\left(R_{c}\right)$ and quantum resistance $\left(R_{q}\right) . R_{c}$ is due to imperfect contact between metal and CNT material and depending on the fabrication process, its value ranges from few Ohms to tens of KiloOhms [36]. $R_{q}$ is due to quantum confinement of charge carriers along interconnect dimensions. It is given as [21]

$$
R_{q}=\frac{h}{4 e^{2} \cdot N}
$$

where $h$ is Planck's constant and $e$ denotes the charge on electron.

$R_{q}$ and $R_{c}$ together represent lumped resistance $\left(R_{\text {lump }}\right)$. $R_{\text {lump }}$ is distributed equally at the two terminal ends in the ESC model and defined as

$$
R_{\text {lump }}^{\prime}=\frac{R_{\text {lump }}}{2}=\frac{R_{c}+R_{q}}{2}
$$

The distributed resistance $\left(R_{\text {dis }}\right)$ in the ESC model represents the scattering resistance per unit length (p.u.l.). The scattering resistance is because of defects, line edge roughness, phonon and acoustic scattering [37]. It is evaluated as

$$
R_{d i s}=\left(\frac{h}{4 e^{2} \cdot N}\right) \cdot\left(\frac{1}{\lambda}\right)
$$

where $\lambda$ denotes the mean free path $(m f p)$ of electron. In SWCNT bundle interconnect, $m f p$ is in range of micrometers. It is given as [22, 38]

$$
\lambda=\frac{10^{3} d}{\left(T / T_{0}\right)-2}
$$

where $T$ is the room temperature and $T_{0}$ is $100 \mathrm{~K}$.

\subsection{Inductance of SWCNT bundle interconnect}

The distributed inductance $\left(L_{d i s}\right)$ in the ESC model is obtained as

$$
L_{d i s}=L_{k}+L_{m}
$$

where $L_{k}$ and $L_{m}$ are kinetic inductance p.u.l. and magnetic inductance p.u.l., respectively. The former is due to the kinetic energy of the mobile charge carriers in the conduction channels. On the other hand, the later occurs due to stored energy of the charge carriers in the conduction channels of the SWCNT bundle interconnect. They are computed as [20, 21]

$$
L_{k}=\left(\frac{h}{2 e^{2} v_{f}}\right)\left(\frac{1}{2 N}\right)
$$

and

$$
L_{m}=\left[\frac{\mu}{2 \pi} \cdot \ln \left(\frac{h_{g}}{d}\right)\right]\left(\frac{1}{N}\right)
$$


where $v_{f}$ represents the Fermi velocity and equals to $8 \times 10^{5} \mathrm{~m} / \mathrm{s}$.

\subsection{Capacitance of SWCNT bundle interconnect}

The distributed capacitance $\left(C_{d i s}\right)$ in the ESC model is evaluated as

$$
C_{d i s}=\frac{C_{q} \cdot C_{e}}{C_{q}+C_{e}}
$$

where $C_{q}$ is the quantum capacitance p.u.l. that arise due to finite density of states at Fermi energy whereas p.u.l. $C_{e}$ accounts for intrinsic electrostatic capacitance of the SWCNTs [39]. $C_{q}$ and $C_{e}$ are given as [21, 38]

$$
\begin{gathered}
C_{q}=\left(\frac{2 e^{2}}{h v_{f}}\right)(2 N) \\
C_{e}=\left(\frac{2 \pi \varepsilon}{\cosh ^{-1}\left(\frac{h_{g}+d / 2}{d}\right)}\right) \cdot\left(N_{w}\right)
\end{gathered}
$$

\subsection{Coupling capacitance and mutual inductance of SWCNT bundle interconnect}

The p.u.l. coupling capacitance $\left(C_{c}\right)$ and mutual inductance $(M)$ between parallel SWCNT bundle interconnect structures are defined as $[20,38]$

$$
C_{c}=\left(\frac{\pi \varepsilon}{\cosh ^{-1}\left(\frac{s}{d}\right)}\right) \cdot\left(N_{t}\right)
$$

$$
\begin{aligned}
M & =\frac{\mu}{2 \pi}\left[\ln \left(\frac{l}{y}+\sqrt{1+\left(\frac{l}{y}\right)^{2}}\right)-\sqrt{1+\left(\frac{y}{l}\right)^{2}}+\frac{y}{l}\right] \\
& \cdot\left(\frac{1}{N_{t}}\right)
\end{aligned}
$$

where $l$ is the length of interconnect and $y$ represents the center-to-center distance between SWCNTs facing each other in a two parallel SWCNT bundle interconnects.

The copper interconnect parasitics viz. $R_{d i s}, L_{d i s}, C_{d i s}, M$ and $C_{c}$ are computed from [40, 41]. Since, contact resistance between substrate and copper is negligible, $R_{\text {lump }}^{\prime}$ is considered as zero for copper interconnect.

\section{FDTD model formulation for copper and SWCNT bundle interconnects using VMS and CMS schemes}

The unified model of $M$-Line coupled CMOS gate driven copper and SWCNT bundle interconnects using VMS and CMS schemes is shown in figure 3. The model is formulated using FDTD technique. The interconnects in figure 3 are characterized using quasi transverse electromagnetic (TEM) model and represented as

$$
\begin{gathered}
\frac{\partial[V]}{\partial z}+[L] \frac{\partial[I]}{\partial t}+[R][I]=0 \\
\frac{\partial[I]}{\partial z}+[C] \frac{\partial[V]}{\partial t}=0
\end{gathered}
$$

where $[V]$ and $[I]$ are $(M \times 1)$ dimensional voltage and current variables along the length of wire and are function of position $(z)$ and time $(t)$. The $(M \times M)$ dimensional p.u.l. interconnect parasitics are defined as

$$
[R]=\left(\begin{array}{ccccc}
R_{d i s 1} & 0 & 0 & \ldots & 0 \\
0 & R_{d i s 2} & 0 & \ldots & 0 \\
\vdots & \vdots & \ddots & \vdots & \vdots \\
0 & 0 & 0 & \cdots & R_{d i s M}
\end{array}\right), \quad[L]=\left(\begin{array}{ccccc}
L_{d i s 1} & M_{1,2} & M_{1,3} & \ldots & M_{1, M} \\
M_{2,1} & L_{d i s 2} & M_{2,3} & \cdots & M_{2, M} \\
\vdots & \vdots & \ddots & \vdots & \vdots \\
M_{M, 1} & M_{M, 2} & M_{M, 3} & \cdots & L_{d i s M}
\end{array}\right)
$$

and

$$
[C]=\left(\begin{array}{ccccc}
C_{d i s 1}+\sum_{i=2}^{M} C_{c 1, i} & -C_{c 1,2} & -C_{c 1,3} & \ldots & -C_{c 1, M} \\
-C_{c 2,1} & C_{d i s 2}+\sum_{i=1,3}^{M} C_{c 2, i} & -C_{c 2,3} & \ldots & -C_{c 2, M} \\
\vdots & \vdots & \ddots & \vdots & \vdots \\
-C_{c M, 1} & -C_{c M, 2} & -C_{c M, 3} & \ldots & C_{d i s M}+\sum_{i=1}^{M-1} C_{c M, i}
\end{array}\right)
$$




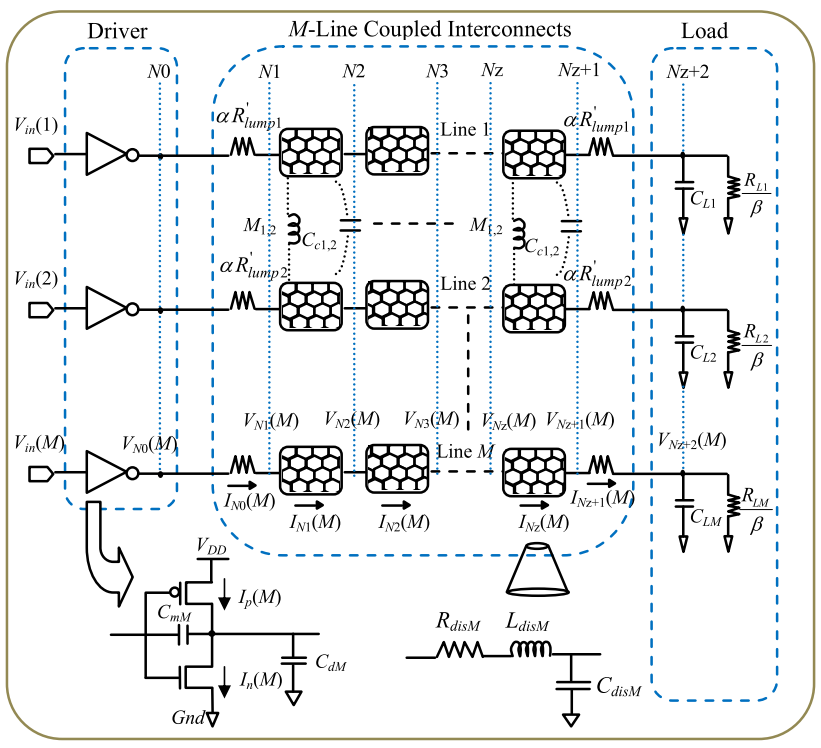

Figure 3. $M$-Line coupled driver interconnect load model.

In figure 3, the input voltage, and NMOS and PMOS drain current are given as

$$
\left[V_{i n}\right]=\left[\begin{array}{c}
V_{i n}(1) \\
V_{i n}(2) \\
\vdots \\
V_{i n}(M)
\end{array}\right],\left[I_{n}\right]=\left[\begin{array}{c}
I_{n}(1) \\
I_{n}(2) \\
\vdots \\
I_{n}(M)
\end{array}\right] \text { and }\left[I_{p}\right]=\left[\begin{array}{c}
I_{p}(1) \\
I_{p}(2) \\
\vdots \\
I_{p}(M)
\end{array}\right]
$$

The $(M \times M)$ dimensional diagonal parameters viz. gate-drain coupling capacitance $\left[C_{m}\right]$, drain diffusion capacitance $\left[C_{d}\right]$, load capacitance $\left[C_{L}\right]$, load resistance $\left[R_{L} / \beta\right]$ and lumped resistance $\left[\alpha R_{\text {lump }}^{\prime}\right]$ are computed as

$$
\begin{aligned}
{\left[C_{d}\right] } & =\operatorname{diag}\left[C_{d 1}, C_{d 2}, \ldots, C_{d M}\right], \\
{\left[C_{m}\right] } & =\operatorname{diag}\left[C_{m 1}, C_{m 2}, \ldots, C_{m M}\right], \\
{\left[C_{L}\right] } & =\operatorname{diag}\left[C_{L 1}, C_{L 2}, \ldots, C_{L M}\right], \\
{\left[R_{L / \beta}\right] } & =1 / \beta \operatorname{diag}\left[R_{L 1}, R_{L 2}, \ldots, R_{L M}\right] \text { and } \\
{\left[\alpha R_{\text {lump }}^{\prime}\right] } & =\alpha \operatorname{diag}\left[R_{\text {lump } 1}^{\prime}, R_{\text {lump } 2}^{\prime}, \ldots, R_{\text {lump }}^{\prime}\right]
\end{aligned}
$$

The formulation of voltage and current variables along the interconnect using FDTD technique is made in the following sub-sections.

\subsection{Formulation of recursive voltage and current variables}

The TEM differential equations in (16)-(17) are discretized in time and position. These are represented as

$$
\left[\begin{array}{l}
\frac{\left[V_{k+1}^{n+2}\right]-\left[V_{k}^{n+2}\right]}{\Delta z}+[L]\left(\frac{\left[I_{k}^{n+3}\right]-\left[I_{k}^{n+1}\right]}{\Delta t}\right) \\
+[R]\left(\frac{\left[I_{k}^{n+3}\right]+\left[I_{k}^{n+1}\right]}{2}\right)
\end{array}\right]=0
$$

and

$$
\frac{\left[I_{k}^{n+1}\right]-\left[I_{k-1}^{n+1}\right]}{\Delta z}+[C] \frac{\left[V_{k}^{n+2}\right]-\left[V_{k}^{n}\right]}{\Delta t}=0
$$

Equations (21)-(22) are simplified as

$$
\left[I_{k}^{n+3}\right]=[B][D]\left[I_{k}^{n+1}\right]+[B]\left(\left[V_{k}^{n+2}\right]-\left[V_{k+1}^{n+2}\right]\right)
$$

where $[B]=\left(\frac{\Delta z}{\Delta t}[L]+\frac{\Delta z}{2}[R]\right)^{-1},[D]=\left(\frac{\Delta z}{\Delta t}[L]-\frac{\Delta z}{2}[R]\right)$, $k=N 1, N 2, N 3, \ldots \ldots, N z$ and $n=0,2,4, \ldots \ldots, T m$. and

$$
\left[V_{k}^{n+2}\right]=\left[V_{k}^{n}\right]+[A]\left(\left[I_{k-1}^{n+1}\right]-\left[I_{k}^{n+1}\right]\right)
$$

where $[A]=\left(\frac{\Delta t}{\Delta z} \cdot \frac{1}{[C]}\right), k=N 2, N 3, N 4, \ldots, N z$ and $n=0,2$, $4, . ., \mathrm{Tm}$.

Equations (23)-(24) represent the recursive expressions to compute current and voltage, respectively along the interconnect length $(l)$. The voltage and current variables are evaluated alternatively at fixed interval of time period of $\Delta t / 2$ and spatial distance of $\Delta z / 2$. This is illustrated in figure $4 . \Delta z$ and $\Delta t$ are constraint by Courant stability condition which is defined as $\Delta t \leq \Delta z / v$ [42]. The voltage and current variables in figure 4 are determined as

$$
\begin{gathered}
{\left[I_{i}^{j}\right]=[I]((i-1 / 2) \Delta z,(j / 2) \Delta t)} \\
{\left[V_{i}^{j}\right]=[V]((i-1) \Delta z,(j / 2) \Delta t)}
\end{gathered}
$$

where $i$ and $j$ are positive integer values.

\subsection{Formulation of near-end boundary condition}

The near-end boundary condition is defined by CMOS driver gate and expressed by voltage and current variables at node $N 0$. The MOS transistors in CMOS gate are characterized by $\mathrm{n}^{\text {th }}$ power law model $[34,43]$. The discretized current at near-end node $N 0$ is evaluated by applying KCL as

$$
\left[I_{N 0}^{n+2}\right]=\left(\begin{array}{l}
{\left[I_{p}^{n+2}\right]-\left[I_{n}^{n+2}\right]+\left[C_{m}\right]\left(\frac{\left[V_{i n}^{n+2}\right]-\left[V_{i n}^{n}\right]}{\Delta t}\right)} \\
-\left[C_{m}+C_{d}\right]\left(\frac{\left[V_{N 0}^{n+2}\right]-\left[V_{N 0}^{n}\right]}{\Delta t}\right)
\end{array}\right)
$$




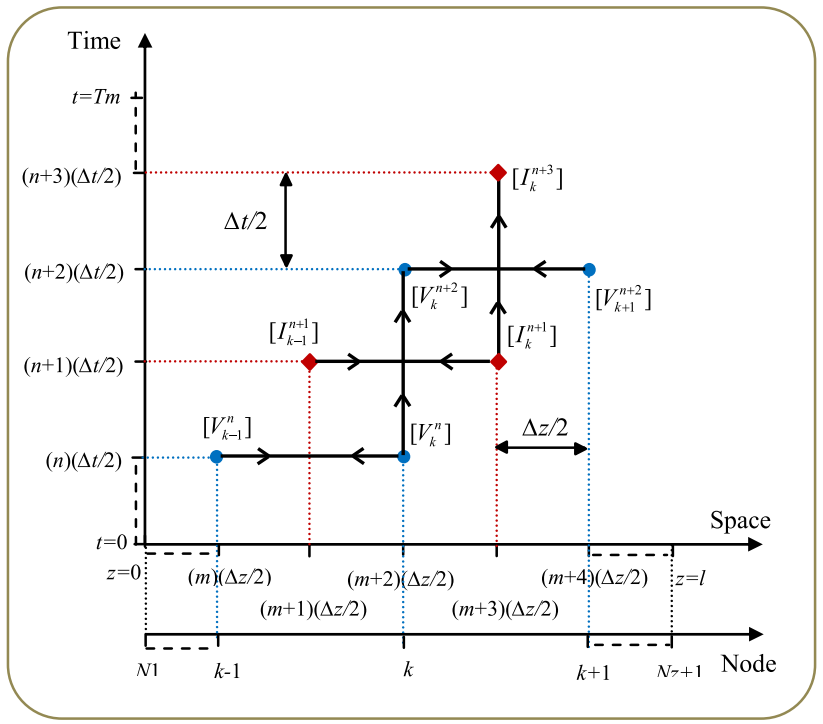

Figure 4. Relationship between spatial, temporal and nodal points.

The discretized voltage at near-end node $N O$ is determined by Ohm's law as

$$
\left[V_{N 0}^{n+2}\right]=\left[V_{N 1}^{n+2}\right]+\alpha\left[R_{\text {lump }}^{\prime}\right] \cdot\left[I_{N 0}^{n+2}\right]
$$

Using (28) in (27), the resultant current at node $N 0$ is further solved as

$$
\left[I_{N 0}^{n+2}\right]=[H]\left(\begin{array}{l}
{\left[I_{p}^{n+2}\right]-\left[I_{n}^{n+2}\right]+\left[C_{m}\right]\left(\frac{\left[V_{i n}^{n+2}\right]-\left[V_{i n}^{n}\right]}{\Delta t}\right)} \\
-\left(\left[C_{m}\right]+\left[C_{d}\right]\right)\left(\frac{\left[V_{N 1}^{n+2}\right]-\left[V_{N 1}^{n}\right]}{\Delta t}\right) \\
+\alpha\left[R_{\text {lump }}^{\prime}\right]\left(\left[C_{m}\right]+\left[C_{d}\right]\right)\left(\frac{\left[I_{N 0}^{n}\right]}{\Delta t}\right)
\end{array}\right)
$$

where

$$
[H]=\left(U+\frac{\alpha\left[R_{\text {lump }}^{\prime}\right]\left(\left[C_{m}\right]+\left[C_{d}\right]\right)}{\Delta t}\right)^{-1}
$$

$\left[V_{N 1}^{n+2}\right]$ in (29) is obtained by substituting $k=1$ in (24). Since, the distance between $\left[I_{N 0}^{n+1}\right]$ and $\left[I_{N 1}^{n+1}\right]$ is $\Delta z / 2, \Delta z$ is replaced by $\Delta z / 2$ in (24) [34]. The resultant expression is

$$
\left[V_{N 1}^{n+2}\right]=\left[V_{N 1}^{n}\right]+2[A]\left(\left[I_{N 0}^{n+1}\right]-\left[I_{N 1}^{n+1}\right]\right)
$$

where

$$
\left[I_{N 0}^{n+1}\right]=\frac{\left[I_{N 0}^{n}\right]+\left[I_{N 0}^{n+2}\right]}{2}
$$

Using (29) and (32) in (31), gives

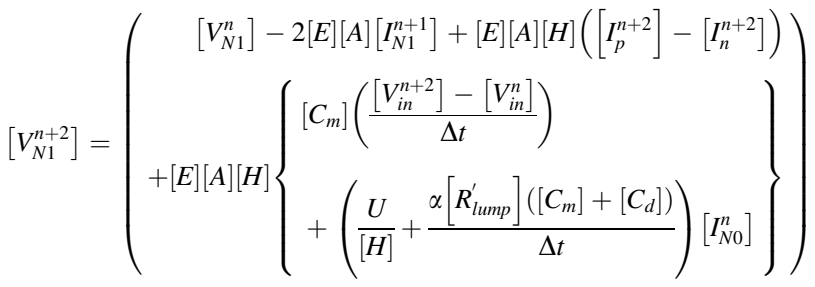

where

$$
[E]=\left(U+\frac{[A][H]\left(\left[C_{m}\right]+\left[C_{d}\right]\right)}{\Delta t}\right)^{-1}
$$

and $U$ is $(M \times M)$ dimensional unity matrix.

Equations (28) and (29) denote the near-end boundary variables.

\subsection{Formulation of far-end boundary condition}

The far-end boundary condition is defined by voltage and current variables at node $\mathrm{Nz}+2$. By applying KCL at node $\mathrm{Nz}+2$, the discretized current at far-end node is obtained as

$$
\left[I_{N z+1}^{n+2}\right]=\left[C_{L}\right]\left(\frac{V_{N z+2}^{n+2}-V_{N z+2}^{n}}{\Delta t}\right)+\frac{\beta\left[V_{N z+2}^{n+2}\right]}{\left[R_{L}\right]}
$$

The voltage at far-end node is formulated as

$$
\left[V_{N z+2}^{n+2}\right]=\left[V_{N z+1}^{n+2}\right]-\alpha\left[R_{\text {lump }}^{\prime}\right] \cdot\left[I_{N z+1}^{n+2}\right]
$$

Equation (36) is used in (35) as

$$
\left[I_{N_{z}+1}^{n+2}\right]=[J]\left(\begin{array}{l}
\left(\frac{\alpha\left[R_{\text {lump }}^{\prime}\right]\left[C_{L}\right]}{\Delta t}\right)\left[I_{N z+1}^{n}\right] \\
+\left(\frac{\left[C_{L}\right]}{\Delta t}+\frac{\beta}{\left[R_{L}\right]}\right)\left[V_{N z+1}^{n+2}\right]-\left(\frac{\left[C_{L}\right]}{\Delta t}\right)\left[V_{N_{z}+1}^{n}\right]
\end{array}\right)
$$

where

$$
[J]=\left(U+\frac{\alpha\left[C_{L}\right]\left[R_{\text {lump }}^{\prime}\right]}{\Delta t}+\frac{\alpha \cdot \beta\left[R_{\text {lump }}^{\prime}\right]}{\left[R_{L}\right]}\right)^{-1}
$$

The voltage at node $N z+1$ is computed using (24). Here also $k$ is substituted as $N z+1$ and $\Delta z$ is replaced by $\Delta z / 2$ [34].

$$
\left[V_{N z+1}^{n+2}\right]=\left[V_{N z+1}^{n}\right]+2[A]\left(\left[I_{N z}^{n+1}\right]-\left[I_{N z+1}^{n+1}\right]\right)
$$

where 


$$
\left[I_{N z+1}^{n+1}\right]=\frac{\left[I_{N z+1}^{n}\right]+\left[I_{N z+1}^{n+2}\right]}{2}
$$

Using (37) and (40), (39) is further modified as

$$
\left[V_{N z+1}^{n+2}\right]=\left(\begin{array}{l}
{[F][G]\left[V_{N z+1}^{n}\right]+2[F][A]\left[I_{N z}^{n+1}\right]} \\
-[F][A]\left(1+\frac{\alpha[J]\left[R_{\text {lump }}^{\prime}\right]\left[C_{L}\right]}{\Delta t}\right)\left[I_{N z+1}^{n}\right]
\end{array}\right)
$$

where

$$
\begin{gathered}
{[F]=\left(U+\frac{[A][J]\left[C_{L}\right]}{\Delta t}+\frac{\beta[A][J]}{\left[R_{L}\right]}\right)^{-1}} \\
{[G]=\left(U+\frac{[A][J]\left[C_{L}\right]}{\Delta t}\right)}
\end{gathered}
$$

The voltage and current variation using far-end boundary condition are given by (36) and (37), respectively. The voltage and current along the interconnect at any time and position can hence be computed by implementing recursive, near-end and far-end conditions.

\section{Results and discussion}

This section evaluates the performance of CMS SWCNT bundle interconnect using the proposed FDTD based model and SPICE [44]. The analysis is carried out for $32 \mathrm{~nm}$ technology node [45]. The efficacy of SWCNT bundle interconnect and CMS scheme is ascertained by comparison with conventional copper interconnect and VMS scheme. The NMOS and PMOS transistors width in CMOS gate are $1 \mu \mathrm{m}$ and $2 \mu \mathrm{m}$, respectively [34]. The diameter of SWCNTs in bundle interconnect is $1 \mathrm{~nm}$.

The SWCNT bundle interconnect parasitics are computed using the formulation presented in section 2. The interconnect dimensions at $32 \mathrm{~nm}$ technology node are presented in table $1[27,45]$. Copper interconnect parasitics are computed from PTM [40]. In VMS scheme, $C_{L}$ is $1 \mathrm{fF}$ and $R_{L}$ is assumed as infinity due to high load impedance [33]. $C_{L}$ and $R_{L}$ for CMS scheme are $0.5 \mathrm{fF}$ and $1 \mathrm{~K} \Omega$, respectively [34].

\subsection{Comparative analysis of copper and SWCNT bundle interconnects using VMS and CMS schemes}

Propagation delay for copper and SWCNT bundle interconnects using VMS and CMS schemes are shown in figure 5. It is analyzed that CMS scheme has lesser propagation delay as compared to VMS scheme for both copper and SWCNT bundle interconnects. This is because of low impedance termination in CMS scheme that reduces voltage swing over the wire. It in turn causes fast charging and discharging of interconnect node capacitances. From the figure, it is also investigated that SWCNT bundle interconnect is faster than its counterpart copper interconnect for both VMS and CMS schemes. For example, at interconnect length of $4500 \mu \mathrm{m}$, SWCNT bundle interconnect has about $9.84 \%$ and $15.61 \%$ lesser propagation delay than copper interconnect for VMS and CMS schemes, respectively. The lower propagation delay in SWCNT bundle interconnect is due to smaller interconnect parasitics and higher number of conduction channels. Further, it is seen that the proposed FDTD model and SPICE results are in good agreement with each other for all the considered cases.

\subsection{Performance analysis of CMS SWCNT bundle interconnect}

The propagation delay in CMS SWCNT bundle interconnect for varying signal transition period and interconnect length is presented in figure 6 . The signal transition period is varied from $20 \mathrm{ps}$ to $100 \mathrm{ps}$ while interconnect length ranges from $500 \mu \mathrm{m}$ to $4500 \mu \mathrm{m}$. It is observed from the figure that with variation in interconnect length, propagation delay increases. This is evident due to higher interconnect parasitics for longer interconnect lengths. However, variation in signal transition periods has lesser impact on propagation delay. For instance, average incremental variation in propagtion delay for variation in interconnect length from $500 \mu \mathrm{m}$ to $4500 \mu \mathrm{m}$ is $94.81 \%$ while variation in signal transition periods from $20 \mathrm{ps}$ to $100 \mathrm{ps}$ causes $13.18 \%$ change in propagation delay.

Figure 7 illustrates the propagation delay for different metallic ratio $\left(P_{m}\right)$ of SWCNTs in a bundle and copper interconnects using proposed FDTD model. From figure 7, it is seen that densely packed SWCNTs $\left(P_{m}=1\right)$ have least propagation delay. This is due to increased number of metallic SWCNTs $(N)$ for higher number of metallic ratio

Table 1. Design parameters for global interconnect at $32 \mathrm{~nm}$ technology node [27, 45].

\begin{tabular}{lcccr}
\hline $\begin{array}{l}\text { Width }(w) \\
(\mathrm{nm})\end{array}$ & $\begin{array}{c}\text { Thickness }(t) \\
(\mathrm{nm})\end{array}$ & $\begin{array}{c}\text { Height }\left(h_{g}\right) \\
(\mathrm{nm})\end{array}$ & $\begin{array}{c}\text { Supply voltage }\left(V_{D D}\right) \\
(\mathrm{V})\end{array}$ & Dielectric constant $\left(\varepsilon_{r}\right)$ \\
\hline 48 & 144 & 86.4 & 0.9 & 2.25 \\
\hline
\end{tabular}




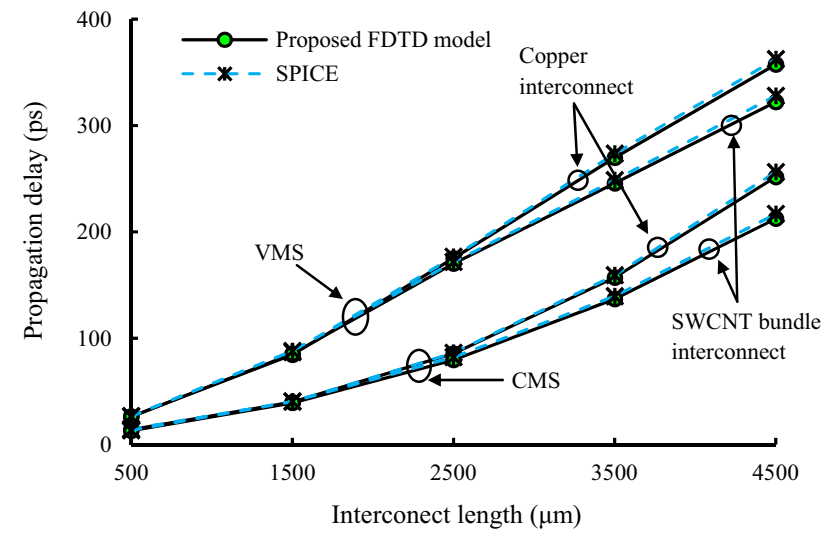

Figure 5. Propagation delay for copper and SWCNT bundle interconnects using VMS and CMS schemes.

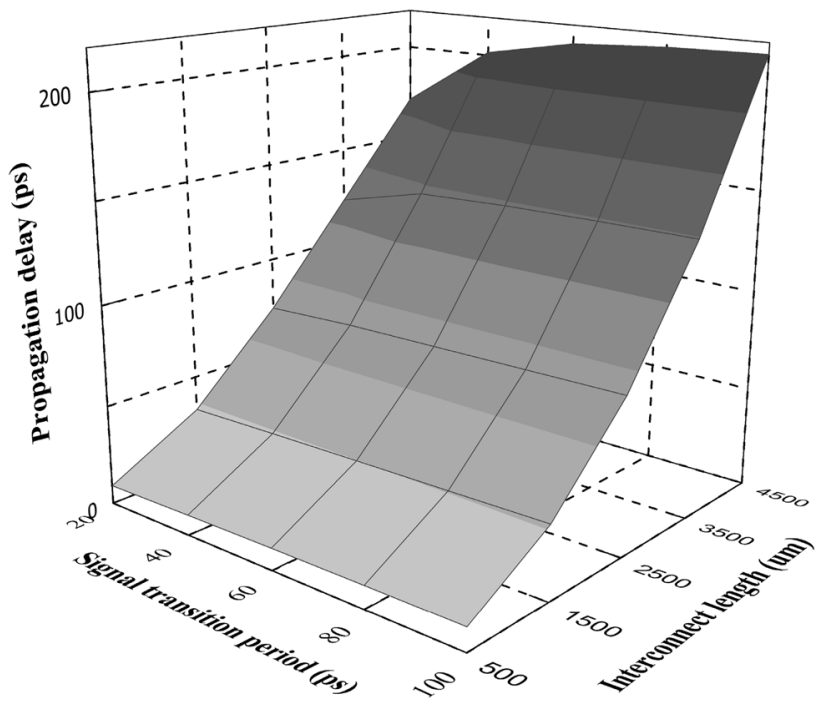

Figure 6. Propagation delay in CMS SWCNT bundle interconnect with variation in signal transition period and interconnect length.

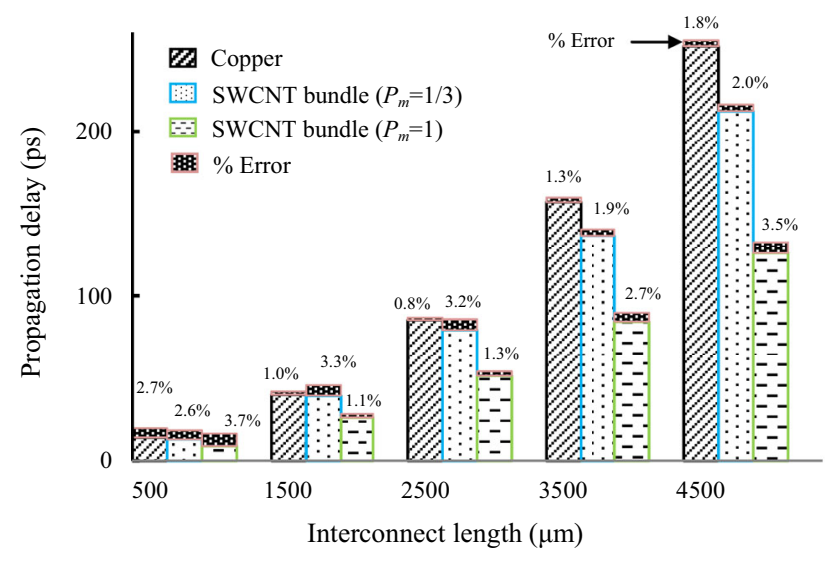

Figure 7. Propagation delay for CMS SWCNT bundle interconnect with variation in SWCNT metallic ratio $\left(P_{m}\right)$. in a bunlde interconnect. The reduction in propagation delay for SWCNTs with $\left(P_{m}=1 / 3\right)$ and SWCNTs with $\left(P_{m}=1\right)$ as compared to copper are $15.61 \%$ and $49.98 \%$, respectively. \%Error in figure 7 denotes the percentage variation between the proposed FDTD and SPICE results. It is observed that maximum percentage error is within $3 \%$ at varying interconnect lengths.

Crosstalk is an important performance metric in on-chip VLSI interconects. It is defined as the occurence of unwanted spurious signals due to capacitive and inductive couplings in coupled interconnect structures [10, 17]. Crosstalk causes delay, glitches, noise at the output and results in mistriggering of logic gates. Crosstalk can be classified as dynamic and functional. In dynamic crosstalk, all the inputs of the system vary either in-phase or out-ofphase with each other while in functional crosstalk, one of the line is quiescent and signals on the other lines vary [24, 25, 34]. Table 2 depicts the crosstalk induced propagation delay for in-phase and out-of-phase switching cases in 3-Line coupled SWCNT bundle interconnects using VMS and CMS schemes. It is analyzed that crosstalk induced propagation delay in out-of-phase is higher than inphase switching case. For instance at interconnect length of $4500 \mu \mathrm{m}$, propagation delay in out-of-phase is about 17.5 and 15.3 times higher than in-phase switching case for SWCNT bundle interconnect using VMS and CMS schemes, respectively. The larger delay in out-of-phase condition is because of higher Miller capacitance effect [46]. It is also analyzed that CMS has lesser impact of crosstalk than VMS scheme. This is evident as crosstalk induced propagation delay in CMS for in-phase and out-ofphase cases have about $63 \%$ and $68 \%$, respectively reduced propagation delay than VMS scheme at interconnect length of $4500 \mu \mathrm{m}$. This justifies that CMS are better for highspeed applications. Further, it is observed that the results obtained using the proposed FDTD model match quite closely with the SPICE results. The average percentage error for different switching cases are within $5 \%$.

Figures 8(a) and 8(b) show the crosstalk induced nosie for different switching cases in 3-Line coupled SWCNT bundle interconnects for VMS and CMS schemes. It is analyzed that both peak overshoot and undershoot noise in figures $8(a)$ and $8(b)$, respectively are lower in CMS scheme. This is because of smaller voltage swing in CMS scheme. From the figure, it is seen that the FDTD model results are at par with SPICE results. The average percentage error between FDTD and SPICE for case(a) and case(b) of figure 8 are $2.1 \%$ and $2.9 \%$, respectively.

\subsection{Variability analysis of CMS SWCNT bundle interconnect}

At nanoscale dimensions, parameter values of interconnects are susceptible to change due to several process and temperature induced variations. With the existing fabrication 
Table 2. Crosstalk induced propagation delay in VMS and CMS SWCNT bundle interconnect.

\begin{tabular}{|c|c|c|c|c|c|c|c|c|c|c|c|c|}
\hline \multirow[b]{4}{*}{$\begin{array}{l}\text { Interconnect } \\
\text { length }(\mu \mathrm{m})\end{array}$} & \multicolumn{12}{|c|}{ Crosstalk induced propagation delay (ps) } \\
\hline & \multicolumn{6}{|c|}{ Voltage-mode signaling } & \multicolumn{6}{|c|}{ Current-mode signaling } \\
\hline & \multicolumn{3}{|c|}{$\begin{array}{c}\text { In-phase switching } \\
\text { (Line } 1-0.9 \text { to } 0 ; \text { Line } 2- \\
0.9 \text { to } 0 ; \text { Line } 3-0.9 \text { to } 0 \text { ) }\end{array}$} & \multicolumn{3}{|c|}{$\begin{array}{l}\text { Out-of-phase switching } \\
\text { (Line } 1-0.9 \text { to } 0 ; \text { Line } 2-0 \\
\text { to } 0.9 ; \text { Line } 3-0.9 \text { to } 0 \text { ) }\end{array}$} & \multicolumn{3}{|c|}{$\begin{array}{c}\text { In-phase switching } \\
\text { (Line } 1-0.9 \text { to } 0 ; \text { Line } 2- \\
0.9 \text { to } 0 ; \text { Line } 3-0.9 \text { to } 0 \text { ) }\end{array}$} & \multicolumn{3}{|c|}{$\begin{array}{c}\text { Out-of-phase switching } \\
\text { Line } 1-0.9 \text { to } 0 ; \text { Line } 2-0 \text { to } \\
0.9 ; \text { Line } 3-0.9 \text { to } 0 \text { ) }\end{array}$} \\
\hline & $\begin{array}{l}\text { Proposed } \\
\text { FDTD } \\
\text { model }\end{array}$ & SPICE & Error & $\begin{array}{l}\text { Proposed } \\
\text { FDTD } \\
\text { model }\end{array}$ & SPICE & Error & $\begin{array}{l}\text { Proposed } \\
\text { FDTD } \\
\text { model }\end{array}$ & SPICE & Error & $\begin{array}{l}\text { Proposed } \\
\text { FDTD } \\
\text { model }\end{array}$ & SPICE & Error \\
\hline 500 & 16.37 & 16.22 & $0.94 \%$ & 140.90 & 143.25 & $1.64 \%$ & 10.81 & 11.01 & $1.88 \%$ & 74.65 & 78.93 & $5.42 \%$ \\
\hline 1500 & 74.63 & 76.49 & $2.43 \%$ & 1113.70 & 1086.30 & $2.52 \%$ & 32.49 & 34.47 & $5.73 \%$ & 432.10 & 447.60 & $3.46 \%$ \\
\hline 2500 & 186.20 & 192.29 & $3.17 \%$ & 3034.40 & 2925.20 & $3.73 \%$ & 72.58 & 76.09 & $4.61 \%$ & 1047.40 & 1105.60 & $5.26 \%$ \\
\hline 3500 & 345.70 & 363.14 & $4.80 \%$ & 5923.10 & 5660 & $4.65 \%$ & 130.10 & 136.03 & $4.36 \%$ & 1967.20 & 2049.60 & $4.02 \%$ \\
\hline 4500 & 555.80 & 588.68 & $5.59 \%$ & 9754.40 & 9290.60 & $4.99 \%$ & 206.20 & 214.17 & $3.72 \%$ & 3156 & 3279 & $3.75 \%$ \\
\hline Avg. error & & $3.38 \%$ & & & $3.51 \%$ & & & $4.06 \%$ & & & $4.38 \%$ & \\
\hline
\end{tabular}

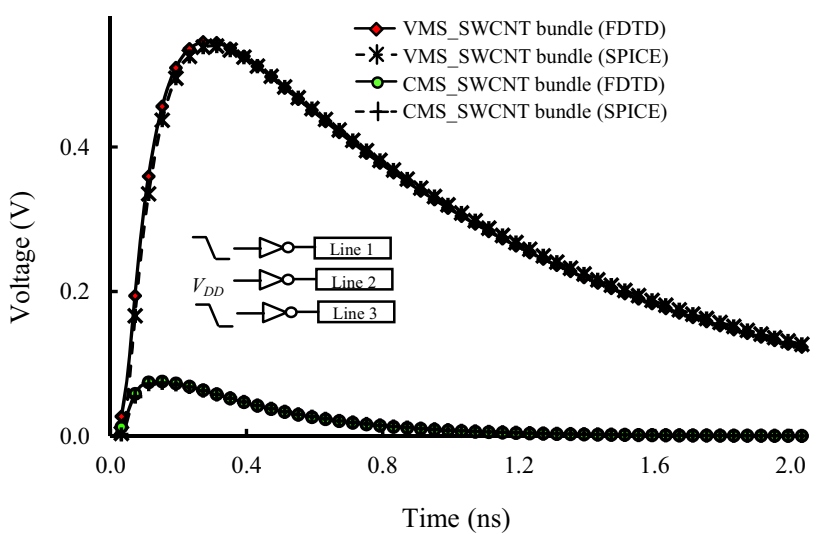

(a)

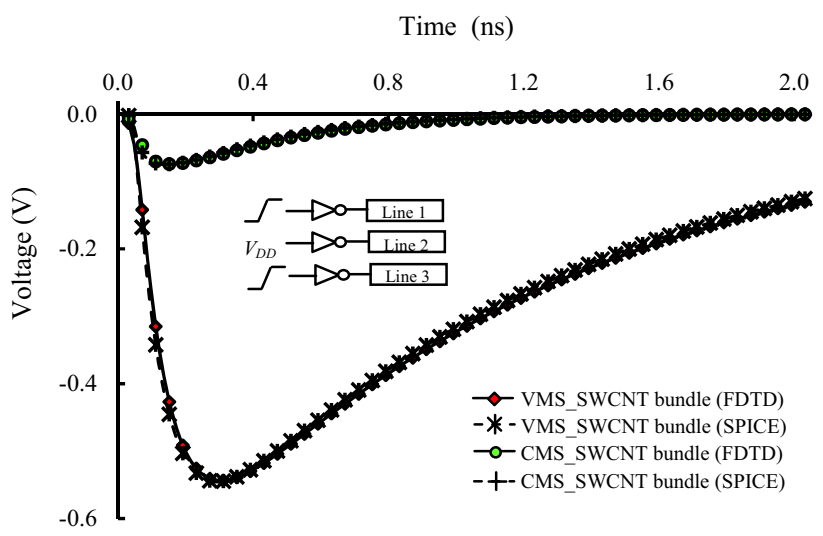

(b)

Figure 8. Transient response at the victim (Line 2) for different switching conditions in 3-Line coupled SWCNT bundle interconnects.

process of CNT structures, the diameter of SWCNTs in bundle interconnect are prone to vary. As a result, number of SWCNTs in the bundle interconnect structure also

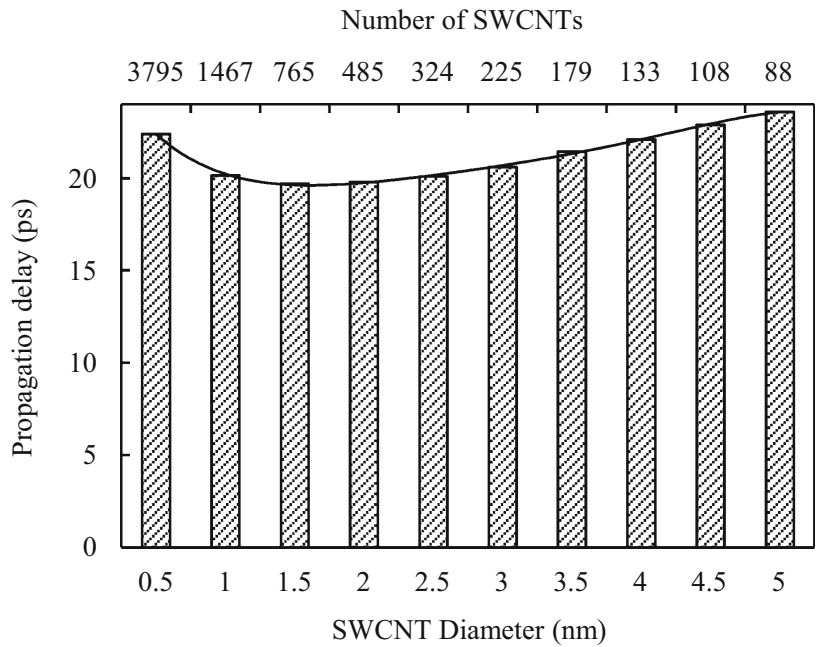

Figure 9. Propagation delay in CMS SWCNT bundle interconnect with varying diameter and number of SWCNTs.

changes. This affects the overall performance of the system. The variation of diameters and their corresponding number of SWCNTs are shown on the primary and secondary horizontal axes in figure 9. Owing to these variations, propagation delay varies and is shown along the vertical axis in the same figure. It is seen that propagation delay is non-linearly correlated with variation of diameter of SWCNTs. Propagation delay is high for smaller and larger SWCNT diameters while it is low for intermediate values. This suggests that controlled fabrication of SWCNT diameter in bundle interconnect structure can lead to smaller propagation delay and henceforth higher performance. In the present analysis, SWCNT diameter of $1.5 \mathrm{~nm}$ is the optimum value that results in the smallest propagation delay of $19.7 \mathrm{ps}$. 
Table 3. Interconnect dimensions and their variation range values.

\begin{tabular}{lcccc}
\hline Interconnect dimensions & Percenatge variaton $(\%)$ & Nominal case & Minimum case & Maximum case \\
\hline Width $(\mathrm{nm})$ & \pm 10 & 48 & 43.2 & 52.8 \\
Space $(\mathrm{nm})$ & \pm 10 & 48 & 43.2 & 52.8 \\
Thickness $(\mathrm{nm})$ & \pm 10 & 144 & 129.6 & 77.76 \\
Height $(\mathrm{nm})$ & \pm 10 & 86.4 & 2.025 \\
Dielectric constant & \pm 10 & 2.25 & 95.04 \\
\hline
\end{tabular}

Table 4. Interconnect parasitic for the varied range of interconnect dimensions.

\begin{tabular}{llll}
\hline $\begin{array}{l}\text { Interconnect } \\
\text { parasitics }\end{array}$ & \multicolumn{1}{c}{$\begin{array}{c}\text { Nominal } \\
\text { case }\end{array}$} & \multicolumn{1}{c}{$\begin{array}{c}\text { Minimum } \\
\text { case }\end{array}$} & $\begin{array}{c}\text { Maximum } \\
\text { case }\end{array}$ \\
\hline Rlump $(\Omega)$ & 6.44 & 8.038 & 5.24 \\
Rdis $(\Omega / m)$ & $4.4 \times 10^{6}$ & $5.49 \times 10^{6}$ & $3.58 \times 10^{6}$ \\
Ldis $(\mathrm{H} / \mathrm{m})$ & $2.75 \times 10^{-6}$ & $3.43 \times 10^{-6}$ & $2.24 \times 10^{-6}$ \\
Cdis $(\mathrm{F} / \mathrm{m})$ & $1.50 \times 10^{-11}$ & $1.36 \times 10^{-11}$ & $1.63 \times 10^{-11}$ \\
\hline
\end{tabular}

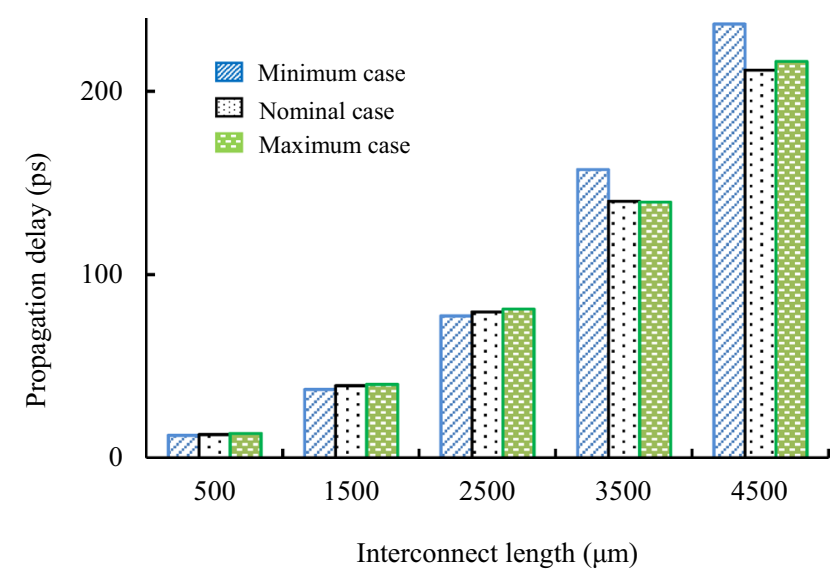

Figure 10. Propagation delay in CMS SWCNT bundle interconnect for nominal, minimum and maximum cases.

The performance of interconnect system with the perturbation of interconnect dimensions have been analyzed. This is essential since due to process induced variations, there exists deviation in the dimensions of interconnects. The interconnect dimensions viz width, thickness, height, spacing between interconnects and dielectric constant are varied by $\pm 10 \%$ [47, 48]. The nominal value of interconnect dimensions at $32 \mathrm{~nm}$ technology node and their variation denoting minimum case and maximum case are shown in table 3 . The interconnect parasitics are computed from these dimensions (as given in table 3) for all the nominal, minimum and maximum cases and are presented in table 4. Figure 10 depicts the propagation delay for the different cases considered. From the figure, it is analyzed that the maximum variation in the propagation delay from the nominal case is nearly $8 \%$ at $4500 \mathrm{~nm}$. It is seen that the effect of variation is very less at shorter interconnect lengths than at longer interconnects.

\section{Conclusion}

This study for the first time analyzes SWCNT bundle interconnect using low voltage swing featured CMS scheme. The performance of CMS SWCNT bundle interconnect is evaluated using numerical technique based FDTD method. The proposed efficient FDTD model is generalized as it can be used for both copper and SWCNT bundle interconnects as well as VMS and CMS signaling schemes. The analyses in the current paper reveal that CMS scheme is faster and lesser prone to crosstalk effects as compared to VMS in SWCNT bundle interconnect. Hence, CMS scheme is an optimum choice for high speed applications in integrated circuit designs. Further, it is analyzed that SWCNT bundle interconnect has lesser propagation delay as compared to copper interconnect for both VMS and CMS schemes. It is also analyzed that the performance of SWCNT bundle interconnect improves as the metallic ratio of SWCNTs in a bundle interconnect increases. This suggests that SWCNT bundle is a prominent material for interconnect applications in nanometer regime. To visualize the effect of process induced variations in interconnect dimensions, variability analysis is performed. It is analyzed that SWCNT with diameter of $1.5 \mathrm{~nm}$ results in optimum performance. From the various analyzes in this contribution, it is observed that the FDTD model and SPICE results are in close agreement with each other. Hence, the proposed FDTD model can be assuredly used for signal integrity analyses for varying interconnect materials and signaling schemes.
List of symbols
$\Delta t \quad$ infinitesimal small time step
$\Delta z \quad$ infinitesimal small distance
$C_{c} \quad$ coupling capacitance per unit length
$C_{d} \quad$ drain diffusion capacitance
$C_{d i s} \quad$ distributed capacitance
$C_{e} \quad$ electrostatic capacitance per unit length
$C_{L} \quad$ load capacitance
$C_{m} \quad$ gate-drain coupling capacitance
$C_{q} \quad$ quantum capacitance per unit length 
$d \quad$ diameter of monolayer SWCNT

$e \quad$ charge on electron

$h \quad$ Planck's constant

$h_{g} \quad$ height above ground plane

I current

$i, j \quad$ positive integer values

int integer value

$l \quad$ length of interconnect

$L_{\text {dis }} \quad$ distributed inductance

$L_{k} \quad$ kinetic inductance per unit length

$L_{m} \quad$ magnetic inductance per unit length

$M \quad$ mutual inductance

$N \quad$ total number of SWCNTs in a bundle

$N_{t} \quad$ number of SWCNTs along the thickness of SWCNT bundle interconnect

$N_{w} \quad$ number of SWCNTs along the width of SWCNT bundle interconnect

Pm metallic ratio

$R c \quad$ contact resistance

$R_{\text {dis }} \quad$ distributed resistance

$R_{L} \quad$ load resistance

$R_{\text {lump }} \quad$ lumped resistance

$R q \quad$ quantum resistance

$s \quad$ distance between two parallel SWCNT bundle interconnect

$t \quad$ thickness of SWCNT bundle interconnect

$T$ room temperature

$t$ time

V voltage

$v_{f} \quad$ Fermi velocity

$w \quad$ width of SWCNT bundle interconnect

$x \quad$ inter-SWCNT distance

$y \quad$ center-to-center distance between SWCNTs facing each other in a two parallel SWCNT bundle interconnects

$z \quad$ position

$\alpha \quad$ parameter for determining interconnect material

$\beta \quad$ parameter for determining signaling scheme

$\lambda \quad$ mean free path

\section{References}

[1] Wong H P and Akinwande D 2011 Carbon nanotube and graphene device physics. Cambridge: Cambridge University Press

[2] Chiariello A G, Miano G, Maffucci, A, Villone F and Zamboni W 2007 Electromagnetic models for metallic carbon nanotube interconnects. Microelectron. Int. Emerald 26(3): 571-585

[3] Cao Q and Rogers J A 2009 Ultrathin films of single-walled carbon nano-materials for electronics and sensors: A review of fundamental and applied aspects. Adv. Mater. 21(1): 29-53

[4] Jorio A, Dresselhaus G and Dresselhaus M S 2008, Carbon nanotubes advanced topics in the synthesis, structure, properties and applications. Berlin: Springer
[5] Misewich J A, Martel R, Avouris P, Tsang J C, Heinze S and Tersoff J 2003 Electrically induced optical emission from a carbon nanotube FET. Science 300(5620): 783-786

[6] Son Y W, Cohen M L and Louie S G 2006 Half metallic graphene nanoribbons. Nature 444(7117): 347-350

[7] Wang N, Tang Z K, Li G, D and Chen J S 2000 Materials science: Single-walled $4 \AA$ carbon nanotube arrays. Nature 408(6808): 50-51

[8] Yu M F, Lourie O, Dyer M J, Moloni K, Kelly T F and Ruoff R S 2000 Strength and breaking mechanism of multiwalled carbon nanotubes under tensile load. Science 287(5453): $637-640$

[9] Lu F, Gu L, Meziani M J, Wang X, Luo P G, Veca L M, Cao L and Sun Y P 2009 Advances in bioapplications of carbon nanotubes. Adv. Mater. 21(2): 139-152

[10] Rai M K, Khanna R and Sarkar S 2014 Crosstalk analysis in CNT bundle interconnects for VLSI applications. IEE J. Trans. Elect. Electron. Eng. 9(4): 391-397

[11] McEuen P L, Fuhrer M S and Park H K 2002 Single-walled carbon nanotube electronics. IEEE Trans. Nanotechnol. 1(1): $78-85$

[12] Javey A 2003 Ballistic carbon nanotube field-effect transistors. Nature 424(6949): 654-657

[13] Novoselov K S, Geim A K, Morozov S V, Jiang D, Zhang Y, Dubonos S V, Grigorieva I V and Firsov A A 2015 Electric field effect in atomically thin carbon films. Science 306(5696): 666-669

[14] Tiang M and Mao J 2015 Modeling and fast simulation of multiwalled carbon nanotube interconnects. IEEE Trans. Electromag. Compat. 57(2): 232-240

[15] Rao P S, Anandatheertha S, Naik G N and Gopalakrishnan 2015 Estimation of mechanical properties of single wall carbon nanotubes using molecular mechanics approach. Sadhana 40(4): 1301-1311

[16] Kuruvilla N and Raina J P 2014 Impact of bundle structure on performance of on-chip CNT interconnects. J. Nanotechnol. 2014: 1-8

[17] Liang F, Wang G and Lin H 2012 Modeling of crosstalk effects in multiwall carbon nanotube interconnects. IEEE Trans. Electromagn. Compat. 54(1): 133-139

[18] Maffucci A, Miano G and Villone F 2008 Performance comparison between metallic carbon nanotube and copper nanointerconnects. IEEE Trans. Adv. Packag. 31(4): 692-699

[19] Naeemi A, Sarvari R and Meindl J D 2005 Performance comparison between carbon nanotube and copper interconnects for gigascale integration (GSI). IEEE Electron Device Lett. 26(2): 84-86

[20] Li H and Banerjee K 2009 High-frequency analysis of carbon nanotube interconnects and implications for on-chip inductor design. IEEE Trans. Electron Devices 56(10): 2202-2214

[21] Sahoo M., Ghosal P and Rahaman H 2014 Performance modeling and analysis of carbon nanotube bundles for future VLSI circuit applications. J. Comput. Electron. 13(3): 673-688

[22] Naeemi A and Meindl J D 2007 Design and performance modeling for single-walled carbon nanotubes as local, semiglobal, and global interconnects in gigascale integrated systems. IEEE Trans. Electron Devices 54(1): 26-37

[23] Zhang K, Tian B, Wang F and Wei J 2012 Crosstalk analysis of carbon nanotube bundle interconnect. Nanoscale Res. Lett. 7(1): $1-5$ 
[24] Pu S N, Lin W Y, Mao J F and Liu Q H 2009 Crosstalk prediction of single- and double-walled carbon-nanotube (SWCNT/DWCNT) bundle interconnects. IEEE Trans. Electron Devices 54(4): 560-568

[25] Das D and Rahaman H 2011 Analysis of crosstalk in singleand multiwall carbon nanotube interconnects and its impact on gate oxide reliability. IEEE Trans. Nanotechnol. 10(6): 1362-1370

[26] Sathyakam P U and Mallick P S 2012 Towards realization of mixed carbon nanotube bundles as VLSI interconnects: A review. Nano Commun. Netw. 3(3): 175-182

[27] Duksh Y S, Kaushik B K and Agarwal R P 2015 FDTD technique based crosstalk analysis of bundled SWCNT interconnects. J. Semicond. 36(5): 055002-055009

[28] Agrawal Y, Chandel R and Dhiman R 2015 High performance current mode receiver design for on-chip VLSI interconnects. Springer In: Proceedings of the international conference on ICA series: Advances in intelligent systems and computing Chapter 54(343): 527-536

[29] Yuan F 2007 CMOS Current mode circuits for data communication. New York: Springer

[30] Chandel R, Sarkar S and Agarwal R P 2005 Transition time considerations in repeater-chains. Microelectron. Int. Emerald 22(3): 39-40

[31] Tuuna S, Nigussie E, Isoaho J and Tenhunen H 2012 Modeling of energy dissipation in RLC current-mode signaling. IEEE Trans. Very Large Scale Integr. Syst. 20(6): 1146-1151

[32] Dave M, Jain M, Baghini M S and Sharma D 2013 A variation tolerant current mode signaling scheme for on-chip interconnects. IEEE Trans. Very Large Scale Integr. Syst. 21(2): 342-353

[33] Bashirullah R, Liu W and Cavin R K 2003 Current-mode signaling in deep submicrometer global interconnects. IEEE Trans. Very Large Scale Integr. Syst. 11(3): 406-417

[34] Agrawal Y and Chandel R 2015 Crosstalk analysis of current-mode signalling-coupled RLC interconnects using FDTD technique. IETE Tech. Rev. 33(2): 1-12

[35] Amore M D, Sarto M S and Tamburrano A 2010 Fast transient analysis of next-generation interconnects based on carbon nanotubes. IEEE Trans. Electromagn. Compat. 52(2): 496-503
[36] Cui J P, Zhao W S, Yin W Y and Hu J 2012 Signal transmission analysis of multilayer graphene nano-ribbon (MLGNR) interconnects. IEEE Trans. Electromagn. Compat. 54(1): 126-132

[37] Chiariello A G, Forestiere C, Miano G and Maffucci A 2013 Scattering properties of carbon nanotubes. Microelectron. Int. Emerald 32(6): 1793-1808

[38] Majumder M K, Kaushik B K and Manhas S K 2014 Analysis of delay and dynamic crosstalk in bundled carbon nanotube interconnects. IEEE Trans. Electromagn. Compat. 56(6): 1666-1673

[39] Fathi D and Forouzandeh B 2009 Time domain analysis of carbon nanotube interconnects based in distributed RLC model. NANO 4(1): 13-21

[40] Predictive Technology Models 2015 available at: http://ptm. asu.edu

[41] Wong S C, Lee G Y and Ma D J 2000 Modeling of interconnect capacitance, delay and crosstalk in VLSI. IEEE Trans. Semicond. Manuf. 13(1): 108-111

[42] Paul C R 1994 Incorporation of terminal constraints in the FDTD analysis of transmission lines. IEEE Trans. Electromagn. Compat. 36(2): 85-91

[43] Sakurai T and Newton A R 1991 A simple MOSFET model for circuit analysis. IEEE Trans. Electron Devices 38(4): 887-894

[44] Tanner EDA tools for SPICE simulation 2015 available at: http://www.tannereda.com

[45] International Technology Roadmap for Semiconductors (ITRS) 2009 available at: http://public.itrs.net

[46] Jiang I, Chang Y W and Jou Y Y 2002 Crosstalk driven interconnect optimization by simultaneous gate and wire sizing. IEEE Trans. Comput. Aided Des. 19(9): 999-1010

[47] Agrawal Y, Chandel R and Dhiman R 2017 Variability analysis of stochastic parameters on the electrical performance of on-chip current-mode interconnect system. IETE J. Res. 63(2): 262-280

[48] Alam N, Kureshi A K, Hasan M and Arslan T 2009 Performance comparison and variability analysis of CNT bundle and copper interconnects. In: IEEE international conference on multimedia, signal processing and communication technologies 169-172 